\title{
On the dependence of the type Ia SNe luminosities on the metallicity of their host galaxies
}

\author{
Manuel E. Moreno-Raya \\ Dpto.de Investigación Básica, C.I.E.M.A.T., Avda. Complutense 40, 28040 Madrid, Spain \\ manuelemilio.moreno@ciemat.es \\ Mercedes Mollá \\ Dpto.de Investigación Básica, C.I.E.M.A.T., Avda. Complutense 40, 28040 Madrid, Spain
}

Ángel R. López-Sánchez

Australian Astronomical Observatory, PO Box 915, North Ryde, NSW 1670, Australia;

Department of Physics and Astronomy, Macquarie University, NSW 2109, Australia

\begin{abstract}
Lluís Galbany
Millennium Institute of Astrophysics MAS, Nuncio Monseñor Sótero Sanz 100, Providencia,7500011 Santiago, Chile; Departamento de Astronomía, Universidad de Chile, Camino El Observatorio 1515, Las Condes, Casilla 36-D, Santiago, Chile.
\end{abstract}

José Manuel Vílchez

Instituto de Astrofísica de Andalucía (CSIC), Apdo. 3004, 18080 Granada, Spain

\section{Aurelio Carnero Rosell}

Observatório Nacional, and LIneA Laboratório Interinstitucional de e-Astronomia, Rua Gal. José Cristino 77 Rio de Janeiro, RJ 20921-400, Brazil;

Inmaculada Domínguez

Departamento de Física Teórica y del Cosmos, Universidad de Granada, 18071 Granada, Spain

\begin{abstract}
The metallicity of the progenitor system producing a type Ia supernova (SN Ia) could play a role in its maximum luminosity, as suggested by theoretical predictions. We present an observational study to investigate if such a relationship there exists. Using the $4.2 \mathrm{~m}$ WHT we have obtained intermediate-resolution spectroscopy data of a sample of 28 local galaxies hosting SNe Ia, for which distances have been derived using methods independent to those based on the own SN Ia parameters. From the emission lines observed in their optical spectrum, we derived the gas-phase oxygen abundance in the region where each SN Ia exploded. Our data show a trend, with a $80 \%$ of chance not to be due to random fluctuation, between SNe Ia absolute magnitudes and the oxygen abundances of the host galaxies, in the sense that luminosities tend to be higher for galaxies with lower metallicities. This result seems like to be in agreement with both the theoretically expected behavior, and with other observational results. This dependence $M_{B}-\mathrm{Z}$ might induce to systematic errors when is not considered in deriving SNe Ia luminosities and then using them to derive cosmological distances.

1
\end{abstract}

Subject headings: distance scale — Supernovae: general — Galaxies: abundances — Galaxies: evolution — Stars: evolution - white dwarfs 


\section{INTRODUCTION}

The Supernova Cosmology is based on the wellknown Hubble Diagram (HD) in which distances of $\mathrm{SNe}$ Ia are represented as a function of their redshifts, $z$, usually determined with high accuracy from $\mathrm{SNe}$ Ia or host galaxies spectra. Distances are derived by means of the distance modulus, $\mu=m-M$. Phillips (1993); Hamuy et al. (1996a b), and Phillips et al. (1999) found a correlation between the properties of the SN Ia light curve (LC) and the absolute magnitude in its maximum for $B, V$ and $I$ bands. Therefore, under the assumption that $\mathrm{SNe}$ Ia are standard-calibrated candles, their absolute magnitude $M$ can be obtained from empirical calibrations based on their observed LCs. Thus, the SNe Ia-based cosmology projects discovered the Universe is in accelerated expansion (Perlmutter et al. 1999; Riess et al. 1998).

However, the SNe Ia methodology is calibrated on local objects, whose host galaxies probably share almost solar abundances 1. But chemical abundances change with redshift (e.g. Lara-López et al. 2009, and references therein) due to chemical evolution of galaxies, therefore the LC calibration might not be the same for chemical abundances which differ very much from solar values. This possible metallicity dependence of the SNe Ia luminosity has been neglected but it might play a role in accurately determining the distances to cosmological SNe Ia. Since the number of SNe Ia detections will extraordinarily increase in the forthcoming surveys (DES2, LSST 3 ), statistical errors will decrease while systematic errors will begin to dominate, limiting the precision of SNe Ia as indicators of extragalactic distances. The metallicity dependence may be one source of systematic errors when using these techniques, being therefore important to quantify its effect.

A dependence of the maximum luminosity of $\mathrm{SNe}$ Ia on the initial metallicity of their progenitors is theoretically predicted. SNe Ia are thought to be thermonuclear explosions of carbon-oxygen white dwarfs (WD) in binary systems, (Whelan \& Iben 1973; Iben \& Tutukov 1984; Webbink 1984). The WD approaches the critical Chandrasekhar mass by accretion from its companion. The maximum luminosity

\footnotetext{
${ }^{1}$ Here we use the terms metallicity or total abundance in metals, $\mathrm{Z}$, (being $\mathrm{X}+\mathrm{Y}+\mathrm{Z}=1$ in mass), and oxygen abundances indistinctly, assuming $\log \left(\mathrm{Z} / \mathrm{Z}_{\odot}\right)=\log (\mathrm{O} / \mathrm{H})-\log (\mathrm{O} / \mathrm{H})_{\odot}, 12+\log (\mathrm{O} / \mathrm{H})_{\odot}=8.69$, and $Z_{\odot}=0.016$ being the solar values Asplund et al. 2009).

${ }^{2} \mathrm{http}: / /$ www.darkenergysurvey.org

${ }^{3} \mathrm{http}: / /$ www.lsst.org/lsst/
}

of a SN Ia depends on the amount of ${ }^{56} \mathrm{Ni}$ synthesized during the explosion (Arnett et al. 1982):

$$
L \propto M\left({ }^{56} \mathrm{Ni}\right) \operatorname{erg~s}^{-1},
$$

Assuming the mass of the exploding WD is constant, the parameter who leads the relation between the LC parameters and its maximum magnitude is the outer layer opacity of the ejected material (Hoeflich \& Khokhlov 1996), which depends on temperature and, thus, on the heating due to the radioactive ${ }^{56} \mathrm{Ni}$ decay. The neutron excess in the exploding $\mathrm{WD}$, which controls the radioactive $\left({ }^{56} \mathrm{Ni}\right)$ to non-radioactive (Fe-peak elements) abundance ratio, depends directly on the initial metallicity of the progenitor. Therefore the maximum luminosity of the SN Ia explosion depends on the initial abundances of $\mathrm{C}, \mathrm{N}, \mathrm{O}$, and $\mathrm{Fe}$ of the WD progenitor (Timmes, Brown, \& Truran 2003; Travaglio, Hillebrandt, \& Reinecke 2005; Podsiadlowski et al. 2006). Timmes, Brown, \& Truran (2003) found a dependence on $\mathrm{Z}$ as $1-0.057 \mathrm{Z} / \mathrm{Z}_{\odot}$. Bravo et al. (2010) computed a series of SNe Ia explosions, finding a stronger dependence on $\mathrm{Z}$ :

$$
M\left({ }^{56} \mathrm{Ni}\right) \sim f(\mathrm{Z})=1-0.075 \frac{\mathrm{Z}}{\mathrm{Z}_{\odot}}
$$

They also explored the dependence of the explosion on the local chemical composition, finding a nonlinear relation :

$$
M\left({ }^{56} N i\right) \sim f(\mathrm{Z})=1-0.18 \frac{\mathrm{Z}}{\mathrm{Z}_{\odot}}\left(1-0.10 \frac{\mathrm{Z}}{\mathrm{Z}_{\odot}}\right)
$$

Since the luminosity decreases with $\mathrm{Z}$ increasing, $\mathrm{SNe}$ Ia located in galaxies with $\mathrm{Z}>\mathrm{Z}_{\odot}$ might be dim$m e r$ than expected as compared to those with $\mathrm{Z} \leq \mathrm{Z}_{\odot}$

The dependence of SNe Ia luminosities on the metallicity was studied by Gallagher et al. (2005), who estimated oxygen elemental abundances by using host-galaxies emission lines, finding most metal-rich galaxies have the faintest SNe Ia. They based their conclusion on the analysis of the HD residuals, implying they used SNe Ia to extract the magnitudes. Gallagher et al. (2008) analyzed spectral absorption indices in early-type galaxies, using theoretical evolutive synthesis models (still not very precise, Sánchez et al. in prep.), also finding a trend between $\mathrm{SNe}$ Ia magnitudes and the metallicity of their stellar populations. These results are in agreement with theoretical predictions. 
Other dependences of SNe Ia magnitudes have been found studying the correlations between the residuals in the HD, as $\mu-\mu_{f i t}$, and the host galaxy characteristics. SNe Ia in massive galaxies result brighter than spected after correcting for their LC widths and colors (see Howell et al. 2009; Neill et al. 2009; Sullivan et al. 2010; Lampeitl et al.2010; Childress et al. 2013; Pan et al.2014; Betoule et al.2014; Moreno-Raya et al. 2015, and references therein for more details). Dividing the galaxy mass range into two groups, the $M_{B}$ of SNe Ia shows a step of $\sim 0.07-0.10 \mathrm{mag} \mathrm{dex}^{-1}$ in the residuals plot (see Childress et al. 2013, mainly Table 2 , where observational trends from different authors are compiled) between both bins.

Following Rigault et al. (2013) and Galbany et al. (2014), our aim is to perform a systematic study to determine if SNe Ia luminosities depend on the local elemental abundances of their host galaxies. We built a sample of nearby galaxies hosting $\mathrm{SNe}$ Ia, selecting objects for which distances were determined using methods different to those based on SN Ia. We then conducted intermediate-resolution long-slit spectroscopic observations of the sample to estimate the oxygen gas-phase abundances and, when possible, derive the local metallicity around the region where $\mathrm{SNe}$ Ia exploded. With that we directly check the possible luminosity-metallicity relationship.

\section{OBSERVATIONS AND OXYGEN ABUN- DANCES}

We have observations for 28 local galaxies hosting SNe Ia with the $4.2 \mathrm{~m}$ William Herschel Telescope (WHT) at El Roque de Los Muchachos Observatory, La Palma, Spain, in two campaigns in December 2011 (9 galaxies) and January 2014 (19 galaxies). We observed more galaxies but they did not show emission lines with sufficient $\mathrm{S} / \mathrm{N}$ ratio to measure their intensities. Therefore by construction, our sample is biased to star-forming galaxies. We used the two arms of the ISIS spectrograph, covering from 3600 to $5200 \AA$ in the blue and from 5850 to $7900 \AA$ in the red, with $0.45 \AA /$ pix and $0.49 \AA /$ pix, respectively. Galaxies were chosen from Neill et al. (2009), selecting objects not in the Hubble flow $(z \leq 0.02)$ and for which distances not based on SNe Ia data are available. Table 1 compiles the details of the observed sample. 89 $\mathrm{H}$ II regions were analyzed. We have many galaxies with oxygen abundances estimates for several regions, for which we determined a metallicity radial gradient

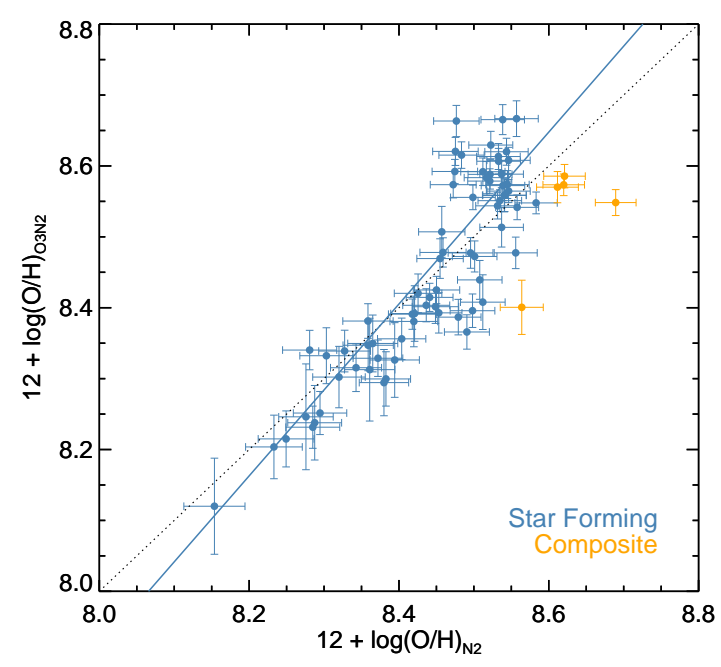

Fig. 1.- Oxygen abundances estimated with the N2 parameter, compared with those obtained using the O3N2 parameter, for the subsample. Blue and orange dots correspond to star-forming and composite regions, respectively. The dashed line is the identity line, while the solid line is a linear fit to the data.

\section{(Moreno-Raya et al. 2015).}

Only $63 \mathrm{H}$ in regions could be classified according to the classical diagnostic diagrams (Baldwin, Phillips \& Terlevich 1981), since the [O III] $\lambda 5007$ was not detected in 26 of them. Other 56 were unambiguously classified as pure star-forming regions; 4 regions are within the Kewley et al. (2001) and Kauffmann et al. (2003) lines in the $\left[\mathrm{O}_{\text {III }}\right] \lambda 5007 / \mathrm{H} \beta$ vs. [ $\left.\mathrm{N}_{\text {II }}\right] \lambda 6583 / \mathrm{H} \alpha$ diagrams, and considered composite objects, still included in our analysis; 3 regions were classified as AGNs and are not longer considered in our analysis. We then define our subsample with 60 star-forming + composite objects.

Values obtained for the 26 objects for which [O III] $\lambda 5007$ is not available are below the low-limit to be defined as AGNs in the $\left[\mathrm{N}_{\mathrm{II}}\right] \lambda 6583 / \mathrm{H} \alpha$ distribution. Hence, we consider these 26 objects as $\mathrm{H}_{\text {II }}$ regions too, and we will use their emission lines to estimate their oxygen abundances. Our final sample has a total of $56+4+26=86 \mathrm{H}$ II regions.

As the faint auroral lines used to compute the electron temperature of the ionized gas (e.g., [O III] 14363) are not detected in any case, we use empirical calibrations (López-Sánchez et al. 2012) to estimate the oxygen abundance. We use $N 2$ and $O 3 N 2$ parameters (Alloin et al. 1979), and the calibrations by 
Table 1: Sample of observed galaxies and their associated SNe Ia. Galaxy names in column 1, galaxy center coordinates RA and DEC in columns 2 and 3, and hosted SNe Ia in column 4, host galaxies magnitudes, $M_{B}$, in column 5 , and the corresponding redshift $z$ in column 6. Distance indicator and SN Ia class, as reddened (R), normal (N) or subluminous (S), are shown in columns 7 and 8., and LC fitters in column 9.

\begin{tabular}{|c|c|c|c|c|c|c|c|c|}
\hline Object & RA & DEC & SN Ia & $\mathrm{M}_{\mathrm{B}}$ & $z$ & Distance indicator* & SN Ia class & LC fitter \\
\hline MGC 021602 & 060434.9 & -123729 & $2003 \mathrm{kf}$ & -19.86 & 0.007388 & $\mathrm{TF}$ & $\mathrm{N}$ & SALT2 \\
\hline NGC 0105 & 002516.6 & +125322 & $1997 \mathrm{cw}$ & -20.98 & 0.017646 & SN Ia & $\mathrm{R}$ & SALT2 \\
\hline NGC 1275 & 031948.1 & +413042 & $2005 \mathrm{mz}$ & -22.65 & 0.017559 & $\mathrm{TF}$ & $\mathrm{S}$ & SALT2 \\
\hline NGC 1309 & 032206.5 & -152400 & $2002 \mathrm{fk}$ & -20.57 & 0.007125 & CEPH \& TF & $\mathrm{N}$ & SALT2 \\
\hline NGC 2935 & 093644.8 & -210741 & $1996 Z$ & -20.69 & 0.007575 & $\mathrm{TF}$ & $\mathrm{R}$ & SALT2 \\
\hline NGC 3021 & 095057.1 & +33 3313 & 1995al & -19.86 & 0.005140 & $\mathrm{CEPH} \& \mathrm{TF}$ & $\mathrm{N}$ & SALT2 \\
\hline M 82 & 095552.7 & +694046 & 2014J & -20.13 & 0.000677 & PNLF & $\mathrm{N}$ & - \\
\hline NGC 3147 & 101653.6 & +732403 & $1997 \mathrm{bq}$ & -22.22 & 0.009346 & $\mathrm{TF}$ & $\mathrm{N}$ & SALT2 \\
\hline NGC 3169 & 101415.0 & +032758 & $2003 \mathrm{cg}$ & -20.42 & 0.004130 & $\mathrm{TF}$ & $\mathrm{R}$ & SALT2 \\
\hline NGC 3368 & 104645.7 & +114912 & 1998bu & -20.96 & 0.002992 & CEPH \& TF & $\mathrm{R}$ & MLCS2k2 \\
\hline NGC 3370 & 104704.0 & +171625 & 1994ae & -19.77 & 0.004266 & CEPH \& TF & $\mathrm{N}$ & MLCS2k2 \\
\hline NGC 3672 & 112502.5 & -094743 & $2007 \mathrm{bm}$ & -20.63 & 0.006211 & $\mathrm{TF}$ & $\mathrm{R}$ & SALT2 \\
\hline NGC 3982 & 115628.1 & +550731 & 1998aq & -19.91 & 0.003699 & CEPH \& TF & $\mathrm{N}$ & MLCS2k2 \\
\hline NGC 4321 & 122254.8 & +154919 & $2006 \mathrm{X}$ & -22.13 & 0.005240 & CEPH \& TF & $\mathrm{R}$ & SALT2 \\
\hline NGC 4501 & 123159.1 & +142513 & $1999 \mathrm{cl}$ & -23.13 & 0.007609 & $\mathrm{TF}$ & $\mathrm{R}$ & SALT2 \\
\hline NGC 4527 & 123408.4 & +023913 & $1991 \mathrm{~T}$ & -21.55 & 0.005791 & CEPH \& TF & $\mathrm{N}$ & MLCS2k2 \\
\hline NGC 4536 & 123427.0 & +021117 & 1981B & -21.85 & 0.006031 & CEPH \& TF & $\mathrm{N}$ & MLCS2k2 \\
\hline NGC 4639 & 124252.4 & +131527 & $1990 \mathrm{~N}$ & -19.24 & 0.003395 & $\mathrm{CEPH} \& \mathrm{TF}$ & $\mathrm{N}$ & MLCS2k2 \\
\hline NGC 5005 & 131056.2 & +370333 & 1996ai & -21.48 & 0.003156 & $\mathrm{TF}$ & $\mathrm{R}$ & SALT2 \\
\hline NGC 5468 & 140634.9 & -052711 & $1999 \mathrm{cp}$ & -20.33 & 0.009480 & $\mathrm{TF}$ & $\mathrm{N}$ & SALT2 \\
\hline NGC 5584 & 142223.8 & -002316 & 2007af & -19.69 & 0.005464 & $\mathrm{CEPH} \& \mathrm{TF}$ & $\mathrm{N}$ & SALT2 \\
\hline UGC 272 & 002749.7 & -011200 & $2005 \mathrm{hk}$ & -19.42 & 0.012993 & $\mathrm{TF}$ & S & MLCS2k2 \\
\hline UGC 3218 & 050043.7 & +621439 & 2006le & -22.17 & 0.017432 & $\mathrm{TF}$ & $\mathrm{N}$ & SALT2 \\
\hline UGC 3576 & 065307.0 & +500203 & $1998 \mathrm{ec}$ & -20.98 & 0.019900 & $\mathrm{TF}$ & $\mathrm{N}$ & SALT2 \\
\hline UGC 3845 & 072642.7 & +470538 & 1997do & -19.95 & 0.010120 & $\mathrm{TF}$ & $\mathrm{N}$ & SALT2 \\
\hline UGC 4195 & 080506.9 & +664659 & 2000ce & -20.71 & 0.016305 & $\mathrm{TF}$ & $\mathrm{R}$ & SALT2 \\
\hline UGC 9391 & 143437.0 & +592016 & 2003du & -17.85 & 0.006384 & $\mathrm{TF}$ & $\mathrm{N}$ & SALT2 \\
\hline UGCA 17 & 012614.4 & -060539 & $1998 \mathrm{dm}$ & -19.86 & 0.006535 & $\mathrm{TF}$ & $\mathrm{R}$ & - \\
\hline
\end{tabular}

Marino et al. 2013, 4 . With the parameter $O 3 N 2$ we obtain $\mathrm{OH}_{O 3 N 2}=12+\log (O / H)_{O 3 N 2}$ for the subsample, and with $N 2$, we have $\mathrm{OH}_{N 2}=12+\log (O / H)_{N 2}$ for the full sample. Figure 1 compares $\mathrm{OH}_{O 3 N 2}$ and $\mathrm{OH}_{N 2}$ for the subsample. A least-squares linear fit yields:

$$
O H_{O 3 N 2}=1.15( \pm 0.09)-1.23( \pm 0.77) \times O H_{N 2},
$$

(correlation coefficient $r=0.88$ ), expression used to convert $\mathrm{OH}_{N 2}$ abundances to $\mathrm{OH}_{O 3 N 2}$ for those 26 regions lacking of $O 3 N 2$ data.

Once $\mathrm{OH}_{O 3 N 2}$ is determined, we assign an oxygen abundance to the region within each galaxy where its $\mathrm{SN}$ Ia was located. For this we use this procedure:

a) In 21 galaxies where several $\mathrm{H}_{\text {II }}$ were detected,

${ }^{4}$ With this calibration (improved using CALIFA data), is difficult to obtain abundances over 8.7 dex. Photoionization models Kewley et al. 2001) overestimate direct abundances around 0.3-0.5 dex (López-Sánchez et al. 2012). we estimate a radial oxygen gradient and then we use it to compute the oxygen abundance which corresponds to the projected galactocentric distance at which the SN Ia exploded.

b) For 7 galaxies for which the previous method cannot be applied (i.e., few $\mathrm{H}$ II regions or unreal gradient), we just select the abundance corresponding to the closest region to the $\mathrm{SN}$ Ia, being the typical distances $\sim 2-3 \mathrm{kpc}$.

A typical difference of $\sim 0.05$ dex, smaller that the oxygen abundance error ( $0.10 \mathrm{dex})$, is found between metallicities derived using gradient or closest region methods, implying their results agree. We finally got the local oxygen abundance for the whole sample of $28 \mathrm{SNe}$ Ia. 

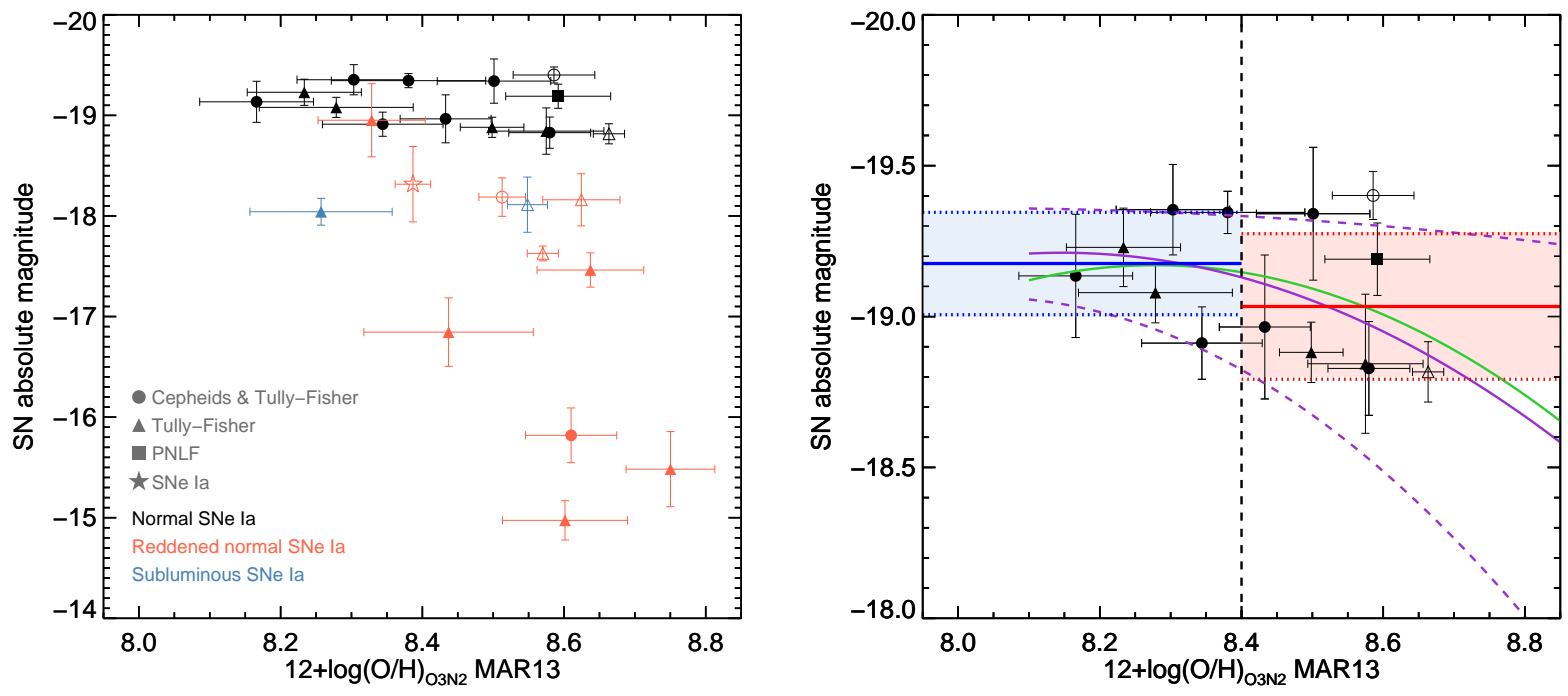

Fig. 2.- Left: SNe Ia absolute magnitudes, $M_{B}$, as a function of oxygen abundances, $\mathrm{OH}_{\mathrm{O} 3 \mathrm{~N} 2}$. Full and open symbols indicate objects with abundances estimated by procedure a) and b), respectively. Triangles, squares and circles indicate the method used to derive distances: Tully-Fisher, PNe Luminosity Function or Tully-Fisher and Cepheids, respectively. The color indicates the type of SN Ia: normal (black), reddened (red), or subluminous (blue). Right: The same plot considering only normal SNe Ia. The green solid line is a second-order polynomial fit to the data. The purple solid line is a second order polynomial fit to four metallicity bins (see text). Short-dashed purple lines represent the 1- $\sigma$ uncertainty to this fit. Blue and red horizontal solid lines provide the averaged value in the low and high metallicity regime, respectively, with their $1-\sigma$ uncertainty shown with the pale blue and red areas.

\section{RESULTS}

\subsection{Estimation of distances and magnitudes}

The distance, $D$, to the galaxies was obtained using the NASA Extragalactic Database, NED5, selecting those independent of the SNe Ia method. The apparent magnitudes, $m_{B}$, of SNe Ia in the maximum of their LCs are from Neill et al. (2009), except for SN2014J taken directly as $M_{B}$ from Marion et al. (2015). We have corrected from the Milky Way extinction 6 using the NED values for B-band, $A_{B}: m_{B, \text { ext }}=m_{B}-A_{B}$. The absolute magnitudes of $\mathrm{SNe}$ Ia were computed applying the usual expression: $M_{B}=m_{B, \text { ext }}-5 \log D+5,(D$ in pc). No standardization technique has been used to obtain the magnitudes.

\section{5 http://hed.ipac.caltech.edu/}

${ }^{6}$ Following Neill et al. (2009) the host galaxy extinction is not accounted for the apparent magnitudes estimation.

\subsection{The relation between the $\mathrm{SN}$ Ia luminosity and the metallicity}

Left panel of Fig. 2 plots SNe Ia absolute magnitudes, $M_{B}$, as a function of the oxygen abundance, $\mathrm{OH}_{\mathrm{O} 3 \mathrm{~N} 2}$. This plot indicates $\mathrm{SNe}$ Ia located in metalrich galaxies are less luminous that the ones in metalpoor galaxies. Right panel of Fig. 2 shows the same only considering normal objects (eliminating reddened and sub-luminous $\mathrm{SNe}$ Ia as explained in Moreno-Raya et al. 2015), where we have fit a second order polynomial function. We have studied the goodness of this last fit via a $\chi^{2}$ test. As errors in both magnitudes and metallicities are relatively large 7 , we have also considered millions of random variations of the values following a Gaussian distribution of the uncertainties in each axis. In each iteration we fit a second-order polynomial to the data and derive the $\chi^{2}$ of the fit. We sought the minimum values of these

\footnotetext{
${ }^{7}$ The average uncertainties of $M_{B}$ and $\mathrm{OH}_{\mathrm{O} 3 \mathrm{~N} 2}$ are $\pm 0.15 \mathrm{mag}$ and \pm 0.08 dex, respectively, while their values ranges are $0.85 \mathrm{mag}$ and 0.50 dex, respectively.
} 
values, which confirm the relationship is satisfied with around a $80 \%$ of probability.

We have also averaged our data in four metallicity bins: $x<8.2,8.2<x<8.4,8.4<x<8.6$ and $x>8.6$, being $x=\mathrm{OH}_{\mathrm{O} 3 \mathrm{~N} 2}$. The purple continuous line plotted in the right panel of Fig. 2 is a second order polynomial fit to the average value obtained for these bins. This fit matches well with that obtained considering all data. Dividing the abundances into a low-metallicity, $\mathrm{OH}_{\mathrm{O} 3 \mathrm{~N} 2}>8.4$, and a high-metallicity, $\mathrm{OH}_{\mathrm{O} 3 \mathrm{~N} 2}<8.4$, regime, we find a difference of $0.14 \pm 0.10 \mathrm{mag}$ in $M_{B}$ (blue and red horizontal lines in the right panel of Fig. 2), with high (low) metallicity galaxies hosting less (more) luminous SNe Ia.

A shift in the magnitudes as due to the metallicity effect over the SNe Ia luminosity is theoretically expected. Considering Eqs. 10 to 3, $L \propto f(\mathrm{Z})$, and $M_{\text {bol }}=-2.5 \log L \sim-2.5 \log [f(Z)]$. Assuming that these equations are also valid in the $B$-band, the difference between the $M_{B}$ of a system with solar abundance and the corresponding $M_{B, Z}$ for any other value of $\mathrm{Z}$ can be computed using the functions $f(\mathrm{Z})$ given in Eq. 1 and 2. Thus, we get a metallicity-dependent magnitude:

$$
M_{B}(\mathrm{Z})=M_{B, Z_{\odot}}+\Delta M_{B}(\mathrm{Z}) \mathrm{mag},
$$

where the term $\Delta M_{B}(\mathrm{Z})$ produces a shift in the expected value $M_{B}$ which corresponds to $Z_{\odot}$.

By using Eqs. 2 and 3 , we derive these two metallicity-dependent relationships:

$$
\Delta M_{B}(\mathrm{Z})=-2.5 \log \left(1-0.075 \frac{\mathrm{Z}}{\mathrm{Z}_{\odot}}\right)-0.0846 \mathrm{mag},
$$

and

$$
\Delta M_{B}(\mathrm{Z})=-2.5 \log \left[1-0.18 \frac{\mathrm{Z}}{\mathrm{Z}_{\odot}}\left(\begin{array}{r}
\left.\left.1-0.10 \frac{\mathrm{Z}}{\mathrm{Z}_{\odot}}\right)\right] \\
-0.191 \mathrm{mag} .
\end{array}\right.\right.
$$

The terms 0.0846 and 0.191 mag have been introduced to normalize these equations to satisfy $\Delta M_{B}\left(\mathrm{Z}_{\odot}\right)=0$. Actually, these values represent the magnitude difference between objects with $Z=0$ and $\mathrm{Z}=\mathrm{Z}_{\odot}$. As we see, the order of magnitude of these variations, $\sim 0.10-0.20 \mathrm{mag}$, agrees with our observational difference of $\sim 0.14 \pm 0.10$ mag between the low- and high-metallicity regimes.

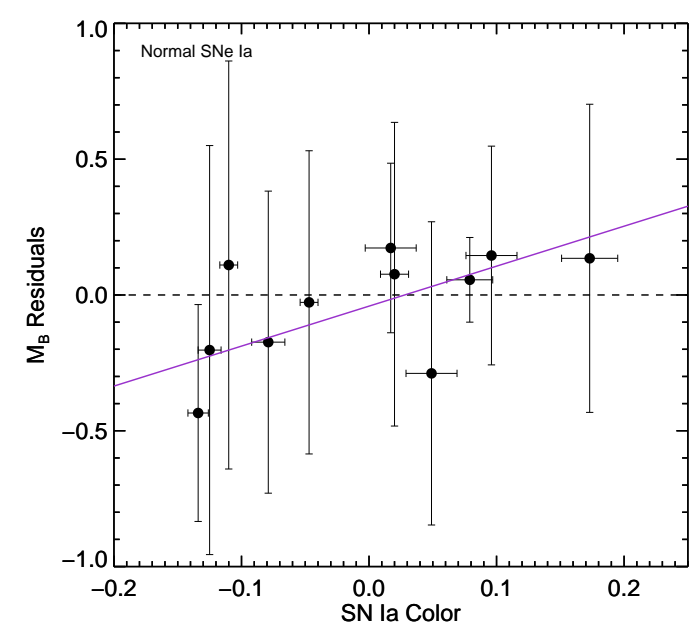

Fig. 3. $-M_{B}$ residuals after subtracting the fit obtained in Fig.2a as a function of the SNe Ia color. The dashed line marks the zero residuals. The solid purple line is the least squares straight line to the dots.

\subsection{The effect of the color in the $M_{B}-\mathrm{Z}$ relation}

This probable metallicity dependence of the luminosity of the SN Ia could be attributed to the color correction, a term already included in the cosmological methods to estimate the distance modulus (and implicitly in the determination of the $M_{B}$ of each SN Ia). Actually, the SNe Ia color shows a dependence on the oxygen abundance and there is also a good correlation between $\mathrm{SNe}$ Ia magnitudes and their colors (see both in Moreno-Raya et al. 2015). However, when we plot Figure 3 with the $M_{B}$ residuals of the fit found in Fig $2 \mathrm{~b}$, as a function of the SNe Ia color, we found, as expected, that there is not a strong correlation, implying that this parameter does not affect very much in the determination of $M_{B}$ for these not reddened objects. A linear fit applied to these points results in:

$$
\Delta M_{B}=-0.04( \pm 0.12)+1.47( \pm 1.42) \times \text { Color }
$$

This fit has a correlation coefficient $r=0.5$, and considering the errors of the fit parameters, a no correlation is equally valid or statistically significant. Therefore, in agreement with Childress et al. (2013), the color dependence is not sufficient to explain the correlation seen in Fig. 2, and a metallicity dependence on $M_{B}$ is still left.

We then conclude that a correlation between the absolute magnitude of SNe Ia, $M_{B}$, and their host-galaxy 

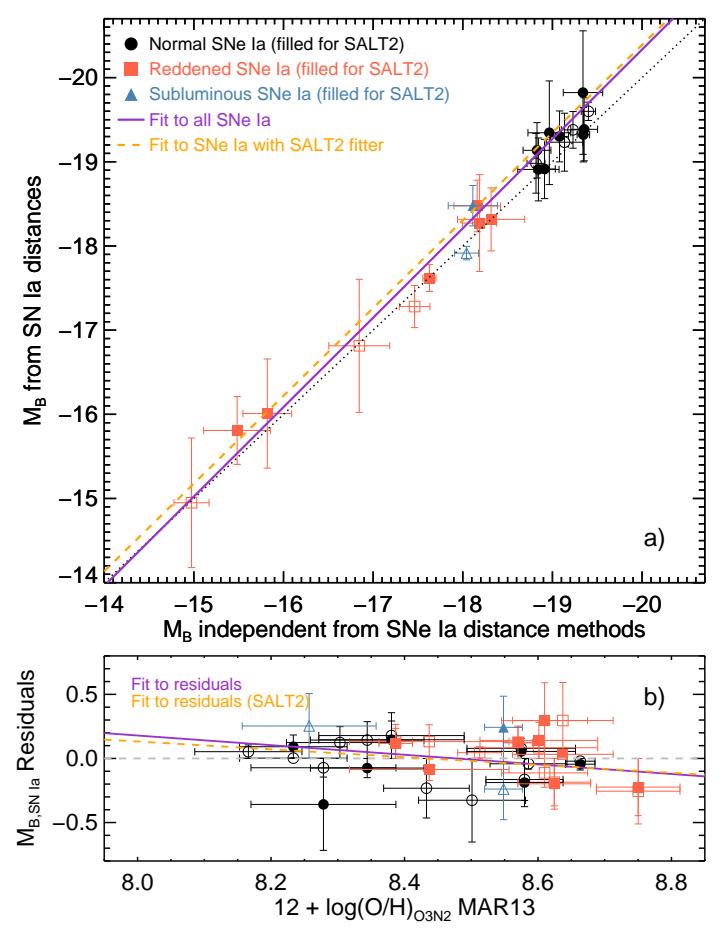

Fig. 4.- a) $M_{B}$ obtained with distances given by the own SN Ia technique vs $M_{B}$ computed with distances estimated with other independent methods. The dotted line is the $x=y$ line, while purple solid and dashed orange lines are linear fits to the data. b) $M_{B}$ residuals from upper panel vs. metallicity.

metallicity seems likely. Metal-rich galaxies host less bright SNe Ia than the metal-poor galaxies. This dependence is not included in the term of color when deriving the distance modulus using $\mathrm{SNe}$ Ia data.

\subsection{Implications of the $M_{B}-\mathrm{Z}$ relation}

If the $M_{B}-\mathrm{Z}$ relation does actually exist, its most important consequence is that the distances of objects obtained by $\mathrm{SNe}$ Ia technique could have a systematic error, as the SNe Ia absolute magnitude has not been accurately estimated.

Figure 4 a shows both magnitudes $M_{B}$ for our SNe Ia sample, as obtained from SN Ia technique distances (taken from NED, prioritizing SALT2 fitter, Guy et al. 2007), and those derived using SNe Ia independent methods, e.g., Cepheids or Tully-Fisher.
A linear fit to SALT2 data yields:

$$
M_{B, \mathrm{SN} \text { Ia }}=0.46( \pm 1.59)+1.04( \pm 0.11) M_{B, \mathrm{SN} \text { Ia ind. }},
$$

(correlation coefficient $r=0.91$ ). Attending to this figure, and although the slope is close to the unity, the $\mathrm{SN}$ Ia technique provides higher luminosities (i.e. higher distances) than the values derived following other methods. That is, luminosities are lower than those predicted by SNe Ia techniques. Figure $4 \mathrm{~b}$ shows the $M_{B, S N I a}$ residuals a a function of oxygen abundance. A linear fit to SALT2 data (orange line) yields:

$$
\Delta M_{B, \mathrm{SN} \text { Ia }}=2.57( \pm 1.95)-0.30( \pm 0.23) \mathrm{OH}_{\mathrm{O} 3 \mathrm{~N} 2}
$$

This would have an impact on the HD of cosmological models. Since magnitudes estimated by the color and LC parameters provide higher luminosities at high metallicities than they really are, some residuals with a positive slope will be induced in the HD when comparing the luminosity of the SN Ia and the oxygen abundance (or any other metallicity proxy, as the stellar mass following the mass-metallicity relation). Such behavior has been in fact observed, since D'Andrea et al. (2011); Childress et al. (2013) and Pan et al. (2014) found that, once splitting their SNe Ia sample in sub-solar and over-solar metallicity, those located in high-metallicity hosts are, on average, $0.10-0.12$ mag brighter than those found in lowmetallicity galaxies. Our results explain this fact with a difference of $0.14 \pm 0.10$ mag between objects at high and low metallicity host galaxy. In summary, it seems SNe I stretch-color-corrected luminosities have a dependence on the properties of their host galaxies, in particular on the oxygen abundance 8 , which could artificially increase this luminosity above the real value.

Therefore, we here suggest to formally include the metallicity-dependence in the determination of the distance modulus, $\mu$, as:

$$
\mu=m_{B}-M_{B}+\alpha x_{1}-\beta c+\gamma \mathrm{Z},
$$

with $\alpha, \beta$ and $\gamma$ being the coefficients for the dependence on stretch, $x_{1}$, color $c$ and metallicity, $Z$, similarly to Lampeitl et al. (2010, Eq.2) and Sullivan et al. (2011), who consider the stellar mass as an extra

\footnotetext{
${ }^{8}$ The SN Ia luminosity has a stronger dependence on the gas-phase metallicity than on the stellar one (see Pan et al. 2014), probably due to the problems of evolutionary synthesis codes, used to determine this last one
} 
parameter. It should minimize the small but quantifiable systematic errors induced by the metallicitydependence of the SNe Ia maximum luminosity.

\section{SUMMARY AND CONCLUSIONS}

- We estimate oxygen abundances of a sample of 28 local star-forming galaxies hosting SNe Ia, in the region where each one exploded, and study the relation with the maximum magnitude $M_{B}$. Data indicate with a $80 \%$ of chance not to be due to random fluctuation, that most metal-rich galaxies seem to host fainter SNe Ia.

- These observational data agree with theoretical predictions from Bravo et al. (2010).

- The existence of such a $M_{B}-\mathrm{Z}$ relation would naturally explain the observational result after correcting for the LC parameters, that brightest $\mathrm{SNe}$ Ia are usually found in metal-rich or massive galaxies. The standard calibration tends to overestimate the maximum luminosities of $\mathrm{SNe}$ Ia located in metal-rich galaxies.

This relation, as obtained here with star-forming galaxies, may indicate the metallicity of the progenitor plays a role in the SN Ia luminosity and hence, in the estimated distances. It could also be that the host galaxy extinction, not considered in this work, correlates with the observed $\mathrm{O} / \mathrm{H}$. This variation with $\mathrm{O} / \mathrm{H}$ would induce systematic errors when using $\mathrm{SNe}$ Ia to derive cosmological distances.

The authors acknowledge the anonymous referee for his/her helpful comments. This work has been partially supported by MINECO-FEDER-grants AYA2010-21887-C04-02 and AYA2011-22460. Support for LG is provided by CONICYT through FONDECYT grant 3140566, and by the Chilean's Millennium Science Initiative through grant IC120009, awarded to The Millennium Institute of Astrophysics, MAS. We thank Eduardo Bravo for their comments to this paper. M.E.M.-R. thanks the hospitality of both the Australian Astronomical Observatory in 2013 and the Departamento de Astronomía of the Universidad de Chile in 2014 during his stays. Based on observations made with the $4.2 \mathrm{~m}$ WHT on the island of La Palma by the Isaac Newton Group of Telescopes in the Spanish observatory of Roque de Los Muchachos of the Instituto de Astrofísica de Canarias. This research has made use of the NASA/IPAC Extragalactic Database (NED), operated by the Jet Propulsion Laboratory, California Institute of Technology, under contract with the National Aeronautics and Space Administration.

\section{REFERENCES}

Alloin D., Collin-Souffrin S., Joly M., Vigroux L., 1979, A\&A, 78, 200

Arnett W. D., 1982, ApJ, 253, 785

Asplund M., Grevesse N., Sauval A. J., Scott P., 2009, ARA\&A, 47, 481

Baldwin J., Phillips M., \& Terlevich R., 1981, PASP, 93, 5

Betoule, M., et al., 2014, A\&A, 568, 22

Bravo E., Domínguez I., Badenes C., Piersanti L., \& Straniero O., 2010, ApJ, 711, L66

Childress M., et al., 2013, ApJ, 770, 108

D’Andrea, C. B., Gupta, R. R., Sako, M., et al. 2011, ApJ, 743, 172

Galbany, L., 2014, A\&A, 572, 38

Gallagher J. S., Garnavich P. M., Caldwell N., Kirshner R. P., Jha S. W., Li W., Ganeshalingam M., Filippenko A. V., 2008, ApJ, 685, 752

Gallagher J. S., Garnavich P. M., Berlind P., Challis P., Jha S., Kirshner R. P., 2005, ApJ, 634, 210

Guy J., et al., 2007, A\&A, 466, 11

Hamuy M., Phillips M. M., Suntzeff N. B., Schommer R. A., Maza J., Aviles R., 1996, AJ, 112, 2391

Hamuy M., Phillips M. M., Suntzeff N. B., Schommer R. A., Maza J., Smith R. C., Lira P., Aviles R., 1996, AJ, 112, 2438

Howell D. A., Sullivan M., Brown E. F., Conley A., Le Borgne D., Hsiao E. Y., Astier P., Balam D., et al. 2009, ApJ, 691, 661

Iben Jr., I. \& Tutukov A. V., 1984,ApJS, 54, 335

Kauffmann G., et al., 2003, MNRAS, 346, 1055

Kelly P. L., Hicken M., Burke D. L., Mandel K. S., \& Kirshner R. P. , 2010, ApJ, 715, 743 
Kewley L. J., Dopita M. A., Sutherland R. S., Heisler C. A., Trevena J., 2001, ApJ, 556, 121

Lampeitl H., Smith M., Nichol R. C., Bassett B., Cinabro D., Dilday B., Foley R. J., Frieman J. A., et al., 2010, ApJ, 722, 566

Lara-López M. A., Cepa J., Bongiovanni A., Pérez García A. M., Castañeda H., Fernández Lorenzo M., Pović M. \& Sánchez-Portal M., 2009, A\&A, $505,529 \mathrm{~L}$

López-Sánchez Á. R., Dopita M.A., Kewley L. J., Zahid H. J., Nicholls D. C., and Scharwächter J., 2012, MNRAS, 426, 2630

Marino R. A., et al., 2013, A\&A, 559, 114

Marion, G. H., Sand, D. J., Hsiao, E. Y., et al. 2015, ApJ, 798, 39

Moreno-Raya M. E., Mollá M., López-Sánchez Á.-R, Galbany L., Vílchez J.M., Domínguez I. \& Carnero A., 2015, MNRAS, in prep.

Neill J. D., Sullivan M., Howell D. A., Conley A., Seibert M., Martin D. C., Barlow T. A., Foster K., et al. 2009, ApJ, 707, 1449

Pan Y.-C., et al., 2014, MNRAS, 438, 1391

Perlmutter S., Aldering G., Goldhaber G., Knop R. A., Nugent P., Castro P. G., Deustua S., Fabbro S., et al. $\&$ The Supernova Cosmology Project, 1999, ApJ, 517,565

Phillips M. M., 1993, ApJ, 413, L105

Phillips M. M., Lira P., Suntzeff N. B., Schommer R. A., Hamuy M., \& Maza J. 1999, AJ, 118, 1766

Podsiadlowski P., Mazzali P. A., Lesaffre P., Wolf C., \& Forster F., 2006, arXiv:astro-ph/0608324

Rigault, M., 2013, A\&A, 560, 66

Riess A. G., Filippenko A. V., Challis P., Clocchiatti A., Diercks A., Garnavich P. M., Gilliland R. L., Hogan C. J., et al. ,1998, AJ, 116, 1009

Hoeflich P., Khokhlov A., 1996, ApJ, 457, 500

Sullivan M., Conley A., Howell D. A., Neill J. D., Astier P., Balland C., Basa S., Carlberg R. G., et al., 2010, MNRAS, 406, 782

Sullivan M., et al., 2011, ApJ, 737, 102
Timmes F. X., Brown E. F., \& Truran J. W., 2003, ApJ, 590, L83

Travaglio C., Hillebrandt W., Reinecke M., 2005, A\&A, 443, 1007

Webbink R. F., 1984, ApJ, 277, 355

Whelan J. \& Iben Jr. I., 1973, ApJ, 186, 1007

This 2-column preprint was prepared with the AAS $\mathrm{LT}_{\mathrm{E}} \mathrm{X}$ macros v5.2. 